\title{
Hole Propagation in the Orbital Compass Models
}

\author{
W. BrzezICKI ${ }^{a, b, *}$, M. DAGHOFER ${ }^{c}$ AND A.M. OLES $\widehat{S}^{a, d}$ \\ ${ }^{a}$ M. Smoluchowski Institute of Physics, Jagiellonian University, prof. S. Łojasiewicza 11, PL-30348, Kraków, Poland \\ ${ }^{b}$ Dipartimento di Fisica "E.R. Caianiello", Universita di Salerno, I-84084 Fisciano (Salerno), Italy \\ ${ }^{c}$ Institut für Theoretische Festkörperphysik, IFW Dresden, D-01171 Dresden, Germany \\ ${ }^{d}$ Max-Planck-Institut für Festkörperforschung, Heisenbergstr. 1, D-70569 Stuttgart, Germany
}

\begin{abstract}
We explore the propagation of a single hole in the generalized quantum compass model which interpolates between fully isotropic antiferromagnetic phase in the Ising model and nematic order of decoupled antiferromagnetic chains for frustrated compass interactions. We observe coherent hole motion due to either interorbital hopping or due to the three-site effective hopping, while quantum spin fluctuations in the ordered background do not play any role.
\end{abstract}

DOI: 10.12693 /APhysPolA.127.263

PACS: 75.10.Jm, 03.65.Ud, 64.70.Tg, 75.25.Dk

\section{Introduction}

Properties of strongly correlated transition metal oxides are determined by effective interactions in form of spin-orbital superexchange, introduced first long ago by Kugel and Khomskii [1]. The spin-orbital interactions have enhanced quantum fluctuations [2] and are characterized by frustration and entanglement [3]. It leads, for instance, to rather cute topological order in an exactly solvable $\mathrm{SU}(2) \otimes X Y$ ring [4]. To understand better the consequences of directional orbital interactions, it is of interest to investigate doped orbital systems [5].

Probably the simplest model that describes orbital-like superexchange is the two-dimensional (2D) orbital compass model (OCM) [6]. The so-called generalized compass model (GCM) introduced later [7] provides a possibility to investigate a second order quantum phase transition (QPT) between the Ising model and generic OCM when frustration increases. The orbital anisotropies are captured in the OCM with different spin components coupled along each bond, $J_{x} \sigma_{i}^{x} \sigma_{j}^{x}$ and $J_{z} \sigma_{i}^{z} \sigma_{j}^{z}$ along $a$ and $b$ axis of the square lattice. Recent interest in this model is motivated by its interdisciplinary character as it plays a role in the variety of phenomena beyond the correlated oxides: (i) it is dual to recently studied models of $p+\mathrm{i} p$ superconducting arrays [8], (ii) it provides an effective description for Josephson arrays of protected qubits [9] realized in recent experiments [10], and (iii) it could also describe polar molecules in optical lattices [11], as well as nitrogen-vacancy centers in a diamond matrix [12].

An exact solution of the one-dimensional (1D) generalized variant of the compass model [13] gives a QPT at $J_{x}=J_{z}$. A similar QCP occurs in the 2D OCM between types of 1D nematic orders: for $J_{x}>J_{z}\left(J_{x}<J_{z}\right)$, antiferromagnetic $(\mathrm{AF})$ chains form along $a(b)$ that are - in the thermodynamic limit - decoupled along $b(a)$.

${ }^{*}$ corresponding author; e-mail: w.brzezicki@uj.edu.pl
It has been shown that the symmetry allows one to reduce the original $L \times L$ compass cluster to a smaller $(L-1) \times(L-1)$ one with modified interactions which made it possible to obtain the full exact spectra and the specific heat for larger clusters [14]. Electron itinerancy has been addressed in the weak-coupling limit at temperatures above the ordering transition [15].

In this paper we will discuss the motion of a single hole in the ordered phases of the GCM, including the nematic phases of the simple OCM. Following [16], we obtain the spectral functions of the itinerant models that reproduce GCM in the strong coupling regime. A great advantage of using the itinerant models is that a variational cluster approach (VCA) can be used to obtain unbiased results for both weak and strong coupling regime. The VCA was introduced to study strongly correlated electrons in models with local interactions $[17,18]$. Since the interactions are here Ising-like, quantum fluctuations are suppressed and the paradigm for hole propagation known from the spin $t-J$ model does no longer apply. This happens for the $t_{2 g}$ electrons in $a b$ planes of $\mathrm{Sr}_{2} \mathrm{VO}_{4}$ where instead holes move mostly via three-site terms [19, 20]. In case of $e_{g}$ electrons, interorbital hopping delocalizes holes within ferromagnetic $\mathrm{LaMnO}_{3}$ planes [21]. In the present case, hole propagation occurs through quantum processes involving the hole itself, rather than those of the ordered background.

\section{Theory}

The 2D GCM with AF interactions $(J>0)$ is

$$
\mathcal{H}_{J}^{\theta}=J \sum_{i}\left\{\bar{\sigma}_{i}(\theta) \bar{\sigma}_{i+a}(\theta)+\bar{\sigma}_{i}(-\theta) \bar{\sigma}_{i+b}(-\theta)\right\}
$$

with $\bar{\sigma}_{i}(\theta)$ being the composed pseudospins

$$
\bar{\sigma}_{i}(\theta)=\cos (\theta / 2) \sigma_{i}^{x}+\sin (\theta / 2) \sigma_{i}^{z},
$$

interpolating between $\sigma_{i}^{x}$ for $\theta=0$ and $\left(\sigma_{i}^{x} \pm \sigma_{i}^{z}\right) / \sqrt{2}$ for $\theta=\pi / 2$ and $\left\{\sigma_{i}^{x}, \sigma_{i}^{z}\right\}$ are $S=1 / 2$ pseudospin operators. $\{i+a(b)\}$ is a shorthand notation for the nearest neighbor of site $i$ along the axis $a(b)$. For $\theta=0$ GCM corresponds to the classical Ising model with $S_{i}^{x}$ components coupled on all the bonds. In the opposite limit $\theta=\pi / 2$ describes 
the OCM in a rotated spin space: bonds along a couple the spin component $\left(S_{i}^{x}+S_{i}^{z}\right)$ and bonds along $b$ the orthogonal one $\left(S_{i}^{x}-S_{i}^{z}\right)$. For $0<\theta<\pi / 2$, the GCM interpolates between Ising and compass models [7]. The rotation of orbital operators (2) provides a convenient way to detect the phase transition between 2D Ising and nematic compass order: In the former, moments lie along $x$ while they lie along either $x+z$ (in the following identified with lattice $\operatorname{axis}^{6} a$ ) or $x-z$ in the latter.

GCM follows from the two-orbital Hubbard model [16],

$$
\begin{aligned}
\mathcal{H}_{t-U} & =t \sum_{i} \sum_{\substack{\mu, \nu=\\
\alpha, \beta}}\left\{A_{\mu \nu} c_{i, \mu}^{\dagger} c_{i+a, \nu}+B_{\mu \nu} c_{i, \mu}^{\dagger} c_{i+b, \nu}+\text { H.c. }\right\} \\
& +U \sum_{i} n_{i, \alpha} n_{i, \beta},
\end{aligned}
$$

at large $U$ and half filling, where $A_{\mu, \nu}$ and $B_{\mu, \nu}$ are hopping matrices in $a, b$ directions between orbitals $\alpha$ and $\beta$. These can be obtained using standard perturbation theory for two neighboring sites as

$$
\begin{aligned}
& A_{\theta}=\frac{1}{\sqrt{2}}\left(\begin{array}{cc}
1+\sin \frac{\theta}{2} & \cos \frac{\theta}{2} \\
\cos \frac{\theta}{2} & 1-\sin \frac{\theta}{2}
\end{array}\right)=\frac{1}{\sqrt{2}}[1+\bar{\sigma}(\theta)], \\
& B_{\theta}=\frac{1}{\sqrt{2}}\left(\begin{array}{cc}
1+\sin \frac{\theta}{2} & -\cos \frac{\theta}{2} \\
\cos \frac{\theta}{2} & 1-\sin \frac{\theta}{2}
\end{array}\right)=\frac{1}{\sqrt{2}}[1-\bar{\sigma}(-\theta)] .
\end{aligned}
$$

The pseudospins $\left\{\sigma_{i}^{x}, \sigma_{i}^{z}\right\}$ are the quadratic forms of the fermions $c_{i}^{\dagger}$, i.e., $\sigma_{i}^{z}=n_{i, \alpha}-n_{i, \beta}, \sigma_{i}^{x}=c_{i, \alpha}^{\dagger} c_{i, \beta}+c_{i, \beta}^{\dagger} c_{i, \alpha}$ and the superexchange is $J=t^{2} / U$. In the small- $U$ regime the properties of the itinerant model of Eq. (3) can be well described by a mean-field (MF) approach [16].

Let us first discuss in more depth the somewhat surprising result that a hole does not couple the AF chains of the OCM. The relevant processes are here the row/column flips along the $x$ or $z$ axis. To see their impact on the itinerant model it is more convenient to look at the OCM in its original basis at site $i,\left\{\tau_{i}^{z}, \tau_{i}^{x}\right\}$. Setting $\tau_{i}^{z, x}=\left(\sigma_{i}^{x} \pm \sigma_{i}^{z}\right) / \sqrt{2}$ we can easily transform GCM at $\theta=\pi / 2$ into OCM with $\tau_{i}^{z} \tau_{i+a}^{z}$ bonds along the $a$ axis and $\tau_{i}^{x} \tau_{i+b}^{x}$ along the $b$ one. Now we can see that OCM commutes with $P_{i}=\prod_{n} \tau_{i+n b}^{z}$ and $Q_{i}=\prod_{n} \tau_{i+n a}^{x}$ operators and the hopping matrices take form of

$$
A_{0}=\left(\begin{array}{ll}
1 & 0 \\
0 & 0
\end{array}\right), \quad B_{0}=\frac{1}{2}\left(\begin{array}{ll}
1 & 1 \\
1 & 1
\end{array}\right) .
$$

To see the action of the row flips in the fermion space, the operator $Q_{i}$ should be first generalized to the case of double and zero occupancy of site $i$. This is achieved by modifying $\tau_{i}^{x}$ as follows, $\tau_{i}^{x} \rightarrow \tilde{\tau}_{i}^{x}=\left(1-n_{i}\right)^{2}+\tau_{i}^{x}$, so that $\left(\tilde{\tau}_{i}^{x}\right)^{2}=1$. Now we can produce new $\tilde{Q}_{i}$ operator in the same way as before and see its action on the fermion operators, which is $\tilde{Q}_{i}\left(c_{j, \alpha(\beta)}\right) \tilde{Q}_{i}=c_{j, \beta(\alpha)}$, for all $c_{j, \mu}$ lying on the line of $\tilde{Q}_{i}$ and unity for the others. Under this change of basis the the interaction part of the $\mathcal{H}_{t-U}$ remains unchanged, i.e., $\tilde{Q}_{i} \mathcal{H}_{U} \tilde{Q}_{i}=U \sum_{i} n_{i, \alpha} n_{i, \beta}$. After a single row-flip $B_{0}$ remains invariant and $A_{0}$ changes as

$$
A_{0}=\left(\begin{array}{ll}
1 & 0 \\
0 & 0
\end{array}\right) \rightarrow\left(\begin{array}{ll}
0 & 0 \\
0 & 1
\end{array}\right) .
$$

This brings us to the conclusion that $\mathcal{H}_{t-U}^{0}$ is covariant under the action of the $\tilde{Q}_{i}$; the exact form of the Hamiltonian changes, but the change is such that the properties of the new Hamiltonian are the same as before - only the orbitals along one line are renamed which is irrelevant for the physics. This is important for the VCA calculation as it allows us to calculate one-particle spectra in one of the nematic ground states, e.g., the AF one, instead of having to average over many of them [16]. For the OCM, results were tested for finite-size effects by changing cluster geometry and size; data presented here are for a $3 \times 4$ cluster.

\section{Results}

In what follows we compare results obtained by the VCA and by MF. A first difference concerns the critical angle $\theta_{c}$ of the QPT from the AF $x$ phase to the AF $a$ one: Whereas the $\mathrm{VCA}$ value $\theta_{c}^{\mathrm{VCA}} \approx 88^{\circ}$ is close to the quasiexact $\theta_{c}^{\mathrm{MERA}} \simeq 84.8^{\circ}$ [7], MF deviates more strongly with $\theta_{c}^{\mathrm{MF}} \approx 68^{\circ}$. While such a discrepancy might suggest the importance of quantum fluctuations within the AF background, we are going to demonstrate that processes related to the hole itself are more important.

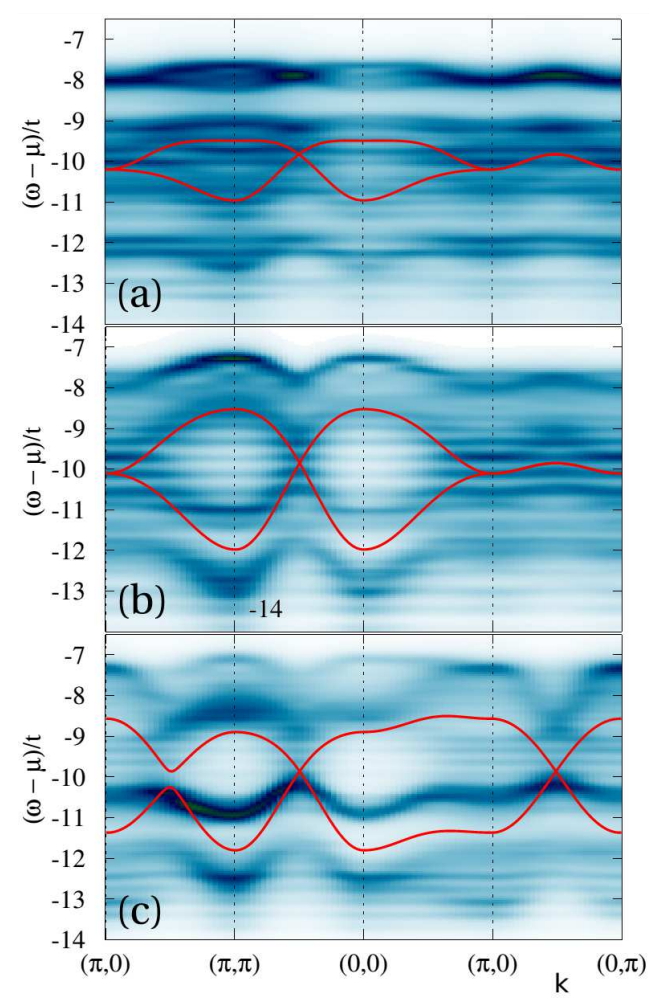

Fig. 1. Spectral functions obtained in the VCA at strong coupling $(U=20 t)$ for the GCM with increasing frustration of interactions at: (a) $\theta=\pi / 6$, (b) $\theta=$ $5 \pi / 12$, and (c) $\theta=89^{\circ}$. The plots (a) and (b) refer to the AF $x$ phase of GCM and plot (c) to the AF $a$ one $\left(\theta_{c}^{\mathrm{VCA}} \approx 88^{\circ}\right)$. Solid lines stand for the MF bands. 
Figure 1 illustrates how the spectral density changes across the QPT from the Ising to nematic order for increasing $\theta$. For $\theta=\pi / 6$, see Fig. 1a, which is very close to the classical AF Ising model, we see a ladder spectrum typical for the $\theta=0$ limit, because at weak quantum fluctuations the hole is confined in a string potential. The two MF bands can naturally not reflect such a ladder spectrum. Nevertheless, MF bands reflect the limited hole mobility and thereby qualitatively reproduce the shape of the topmost VCA band.

For $\theta=5 \pi / 12$, see Fig. 1b, the bands become significantly more dispersive, especially the ones on the top. The shape of the topmost band continues to be qualitatively well reproduced by the MF and this band is the sharpest feature seen in the spectral function at $\theta=$ $5 \pi / 12$. We observe that the bands predicted by MF repel each other in the VCA and new features emerge at the intermediate energies, with rather incoherent weight. Similarly to the generic OCM case, see Fig. 1c in [16], bands are most dispersive along the direction $(0,0) \rightarrow(\pi, \pi)$. Since the ground state is still Ising ordered (AF $x$ phase), the increased dispersion, especially of the rather coherent topmost band, is here not primarily driven by quantum fluctuations. Instead, interorbital hopping is now significant, see Eqs. (4) and (5), which allows the hole to propagate, similar to the case of a hole in $e_{g}$ orbital order [21].

Finally, in Fig. 1c we show the spectral function at $\theta>\theta_{c}$ in the AF $a$ nematic order $\left(\theta=89^{\circ}\right)$. The bottom band is seen as a coherent feature which roughly agrees with the MF prediction, but is much less dispersive. The upper band cannot be identified so easily, even though the features around $\boldsymbol{k}=(\pi / 2, \pi / 2)$ resemble the MF bands. Strong coupling differences to the MF bands are on one hand the incoherent weight and on the other the separation of bottom and top bands. One finds that the MF bands do not really cross at $\boldsymbol{k}=(\pi / 2, \pi / 2)$, but they remain very close to each other. In the $\mathrm{VCA}$, they are better separated, suggesting a strong effective interaction at this value of $\boldsymbol{k}$ that cannot be captured by a simple MF approach. A distinct feature observed in Fig. 1c is a rather coherent band in the middle of the spectrum, absent in the MF approach. It seems to strongly repel the two bands at the top and bottom of the spectrum, thus making them flatter and widening the overall spectrum. We have shown that three-site hopping is the mechanism responsible for the observed dispersion of this additional band [16].

\section{Summary}

We have seen that the coherent motion of a single hole (present for any $\theta>0$ ) is due to: (i) interorbital hopping in the AF phase, and (ii) three-site hopping for the nematic order. MF cannot fully describe either case, it misses the ladder spectrum due to the string potential (AF order) and the three-site hopping (nematic order). In both cases, the hole motion is thus due to the quantum fluctuations caused by the hole itself rather than by the fluctuations of the ordered background.

\section{Acknowledgments}

W.B. acknowledges the kind hospitality of the Leibniz Institute for Solid State and Materials Research in Dresden and the support of the Foundation for Polish Science (FNP) within the START program. W.B. and A.M.O. acknowledge support by the Polish National Science Center (NCN) under Project No. 2012/04/A/ST3/00331. M.D. thanks Deutsche Forschungsgemeinschaft (grant No. DA 1235/1-1 under Emmy-Noether Program) for support.

\section{References}

[1] K.I. Kugel, D.I. Khomskii, JETP 37, 725 (1973); Sov. Phys. Usp. 25, 231 (1982).

[2] G. Khaliullin, Prog. Theor. Phys. Suppl. 160, 155 (2005).

[3] A.M. Oleś, J. Phys. Condens. Matter 24, 313201 (2012); Acta Phys. Pol. A 127, 163 (2015).

[4] W. Brzezicki, J. Dziarmaga, A.M. Oleś, Phys. Rev. Lett. 112, 117204 (2014).

[5] P. Wróbel, A.M. Oleś, Phys. Rev. Lett. 104, 206401 (2010); M. Daghofer, A.M. Oleś, Acta Phys. Pol. A 111, 497 (2007).

[6] Z. Nussinov, J. van den Brink, Rev. Mod. Phys. 87, 1 (2015).

[7] L. Cincio, J. Dziarmaga, A.M. Oleś, Phys. Rev. B 82, 104416 (2010).

[8] Z. Nussinov, E. Fradkin, Phys. Rev. B 71, 195120 (2005).

[9] B. Douçot, M.V. Feigel'man, L.B. Ioffe, A.S. Ioselevich, Phys. Rev. B 71, 024505 (2005).

[10] S. Gladchenko, D. Olaya, E. Dupont-Ferrier, B. Douçot, L.B. Ioffe, M.E. Gershenson, Nature Phys. 5, 48 (2009).

[11] P. Milman, W. Maineult, S. Guibal, L. Guidoni, B. Douçot, L. Ioffe, T. Coudreau, Phys. Rev. Lett. 99, 020503 (2007).

[12] F. Trousselet, A.M. Oleś, P. Horsch, Europhys. Lett. 91, 40005 (2010); Phys. Rev. B 86, 134412 (2012).

[13] W. Brzezicki, J. Dziarmaga, A.M. Oleś, Phys. Rev. B 75, 134415 (2007); W. Brzezicki, A.M. Oleś, Acta Phys. Pol. A 115, 162 (2009).

[14] W. Brzezicki, A.M. Oleś, Phys. Rev. B 82, 060401 (2010); Phys. Rev. B 87, 214421 (2013).

[15] J. Nusu, S. Ishihara, Europhys. Lett. 97, 27002 (2012).

[16] W. Brzezicki, M. Daghofer, A.M. Oleś, Phys. Rev. B 89, 024417 (2014).

[17] C. Dahnken, M. Aichhorn, W. Hanke, E. Arrigoni, M. Potthoff, Phys. Rev. B 70, 245110 (2004).

[18] M. Potthoff, M. Aichhorn, C. Dahnken, Phys. Rev. Lett. 91, 206402 (2003).

[19] M. Daghofer, K. Wohlfeld, A.M. Oleś, E. Arrigoni, P. Horsch, Phys. Rev. Lett. 100, 066403 (2008).

[20] K. Wohlfeld, M. Daghofer, A.M. Oleś, P. Horsch, Phys. Rev. B 78, 214423 (2008).

[21] J. van den Brink, P. Horsch, A.M. Oleś, Phys. Rev. Lett. 85, 5174 (2000). 